Supporting Information

\title{
Failure Mechanisms in Vertically Aligned Dense
}

Nanowire Arrays

Rebecca A. Gallivan*, Julia R. Greer

Division of Engineering and Applied Science, California Institute of Technology, Pasadena, California 91125, United States 


\section{Hydrothermal Growth Substrate and Apparatus}

In this creating the substrates for $\mathrm{ZnO}$ nanowire bundle growth, a $10 \mathrm{~nm}$ platinum adhesion layer and $100 \mathrm{~nm}$ gold layer is deposited via e-beam evaporation (Lesker Labline) onto a silicon substrate. Next, a $1.5 \mu \mathrm{m}$ thick poly(methyl methacrylate) resist (950 PMMA A9) is spin-coated onto the substrate and patterned into an array of holes via e-beam lithography (Raith EBPG 5000+). This holey PMMA coating then acts as a template which allows nanowire growth only in the exposed cylindrical holes, resulting in the bundles. The substrate is then loaded into the cathode side of a custom electrolysis holder (Figure SI1). The holder provides electrical connection to the gold film through a platinum wire. A platinum mesh acts as the counter electrode.

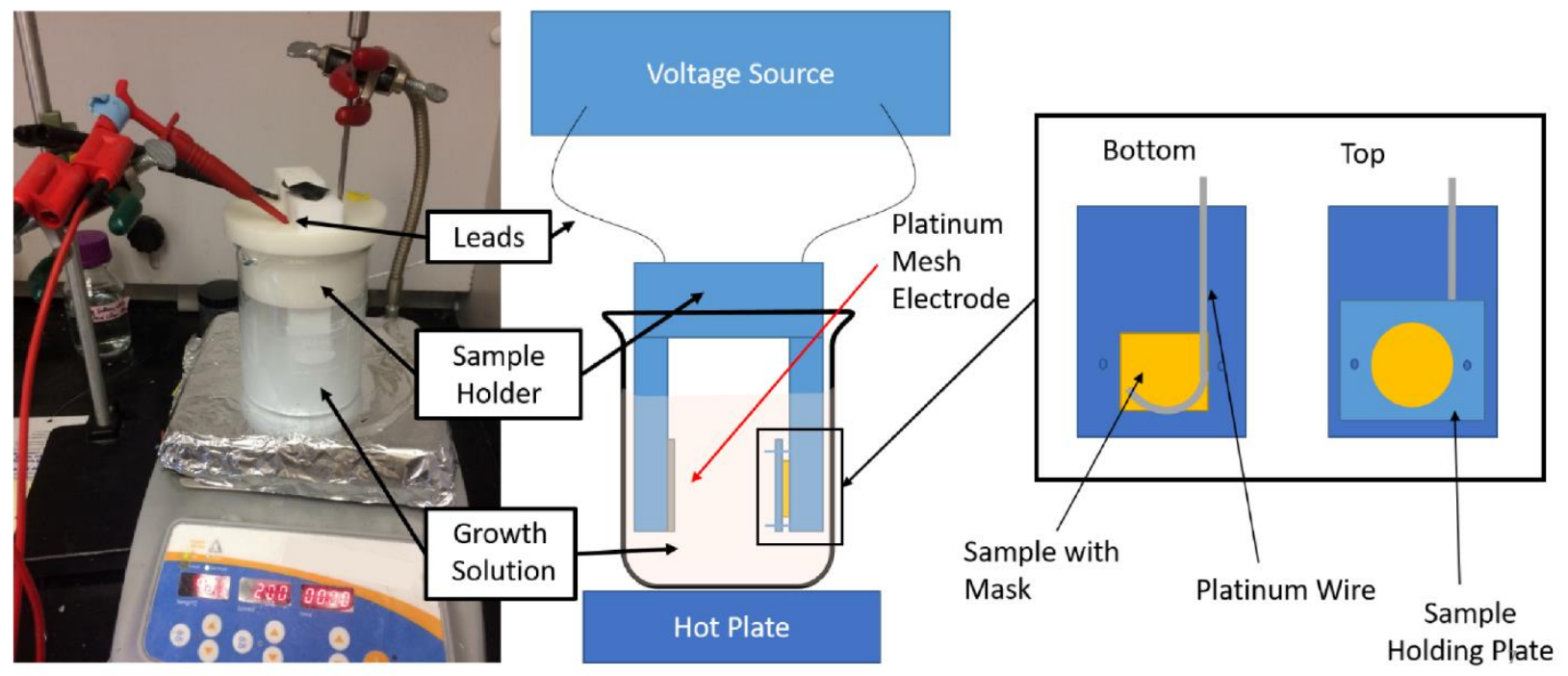

Figure SI1: Hydrothermal growth apparatus and corresponding schematic with custom chip holder. Demonstrates method by which gold coated substrate is submerged into growth solution and connected to electrical source. 


\section{TEM Sample Preparation}

The top surface of the bundle is protected with a $100 \mathrm{~nm}$-thick layer of platinum (Pt) deposited via a Gas Injection System (GIS) using an electron beam in an FEI Versa DualBeam SEM followed by a $400 \mathrm{~nm}$-thick layer of Ga+ FIB-deposited Pt in the same chamber. Next, a Ga+ ion beam is used to carve out $5 \mu \mathrm{m} \times 3 \mu \mathrm{m}$ trenches into the substrate forming a U-cut to free the silicon base from the rest of the substrate. Using a tungsten needle (EZlift program), the detached silicon base with the nanowire bundle on the top surface is transferred and glued via FIB-deposited Pt to a copper halfmoon grid with the long axis of the bundle parallel aligned with the grid post using the same GIS Pt deposition. After detaching the tungsten needle, a series of cuts are made with a decreasing $\mathrm{Ga}+$ voltage/current $(30 \mathrm{kV}, 100 \mathrm{pA} ; 30 \mathrm{kV}, 10 \mathrm{pA} ; 16 \mathrm{kV} 23 \mathrm{pA})$ are used to progressively thin the cross-section of the bundle structure to $<100 \mathrm{~nm}$ for TEM imaging.

3. Tilt Angles of Nanowires

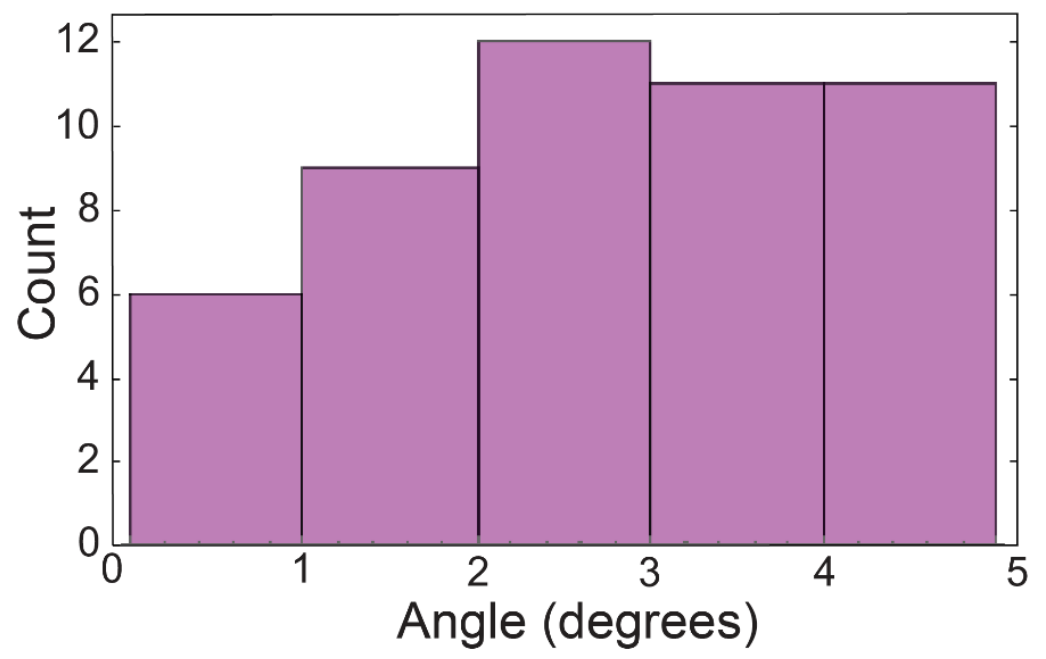

Figure SI3: Histogram showing the relatively uniform distribution of tilt angles measured from as fabricated bundle samples with a mean of $2.8^{\circ}+/-1.2^{\circ}$. 
4. Burst Initiation Stress

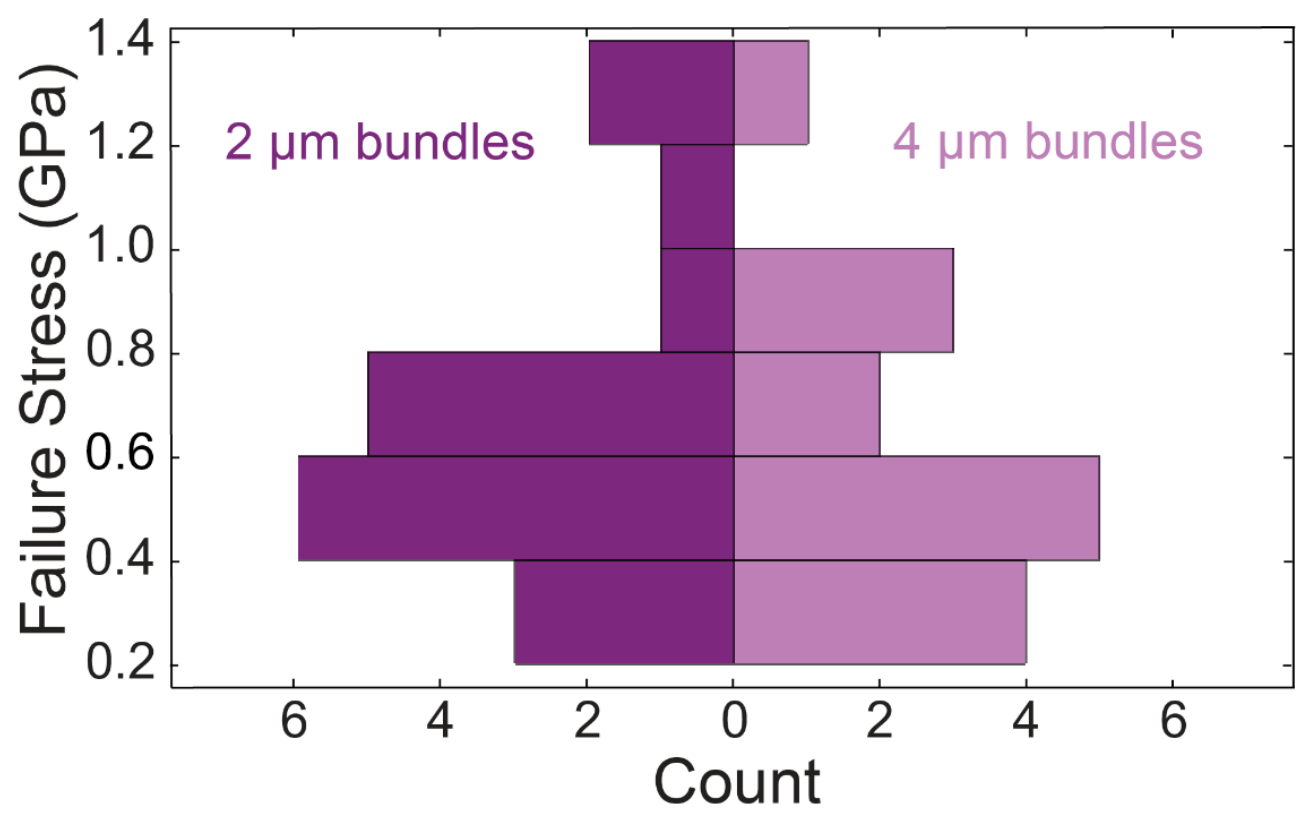

Figure SI4: Histogram compares the distribution of stress associated with initial burst event for 2 $\mu \mathrm{m}$ (left) and $4 \mu \mathrm{m}$ (right) bundles. Distributions show similar range (values from 0.3-1.3 GPa) and mean values $(0.64+/-0.31$ and $0.58+/-0.27 \mathrm{GPa}$ respectively).

\section{Euler Buckling of Nanowires}

Assuming a general column form with a hexagonal cross-section for the individual nanowires, the standard Euler Buckling relationship applies:

$$
\sigma_{\text {buckle }} \geq \frac{\pi^{2} E}{2 k^{2}(l / r)^{2}}
$$

With $l$ being the height, $r$ being the radius, $k$ being the end condition factor (i.e. friction condition), and $E$ the elastic modulus in the direction of loading. The aspect ratio of the nanowires in this 
experiment lies between 26 and 27. We assume a fixed based condition due to surface bonding of the nanowires to the substrate. The interaction between the top surface of the nanowires and the indenter is best described by a free rotation condition $(\mathrm{k}=0.7)$ based on SEM images during insitu compression. Therefore, the critical Euler Buckling stress for these nanowires is $2 \mathrm{GPa}$. To highlight the range of values that could possibly be seen in our system, we include calculations for both fixed $(\mathrm{k}=0.5)$ and free translation $(\mathrm{k}=1.0)$ friction conditions at the indenter-nanowire interface as demonstrated in Figure SI5. The figure highlights the region of our observed nanowires as well as the aspect ratios required for buckling to occur in the range of failure initiation stresses.

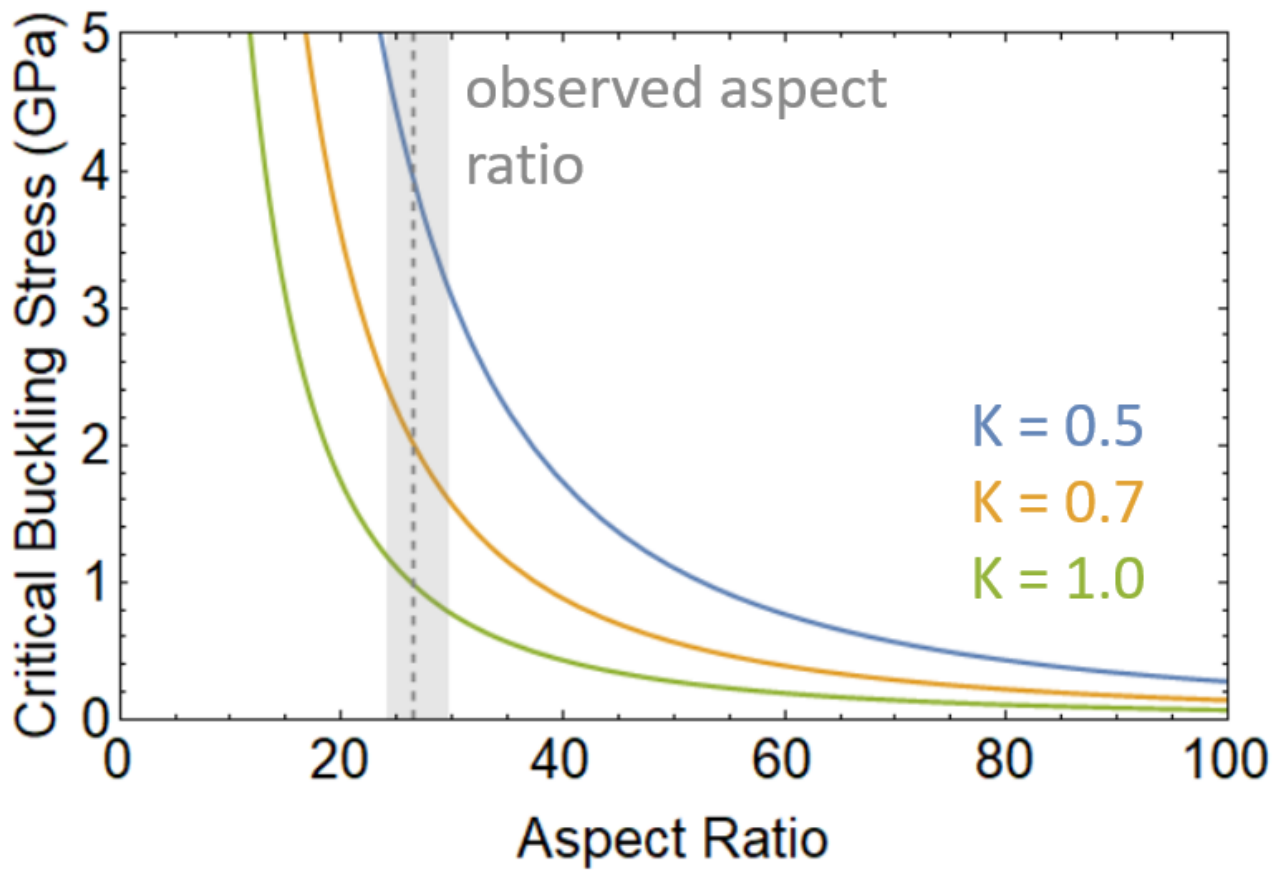

Figure SI5: Critical buckling stress for an unsupported hexagonal $\mathrm{ZnO}$ nanowire of particular aspect ratio given a fixed base and fixed $(\mathrm{k}=0.5)$, pinned $(\mathrm{k}-0.7)$, or free $(\mathrm{k}=1.0)$ boundary condition. Grey area reflects experimentally average $+/$ - a standard deviation in observed dimensions of the nanowires. 
Table SI5 further identifies the specific aspect ratios require for critical buckling failure to occur at the stress values associated with our onset of nanowire interfacial splitting. Values bolded indicate conditions in which buckling would likely be a dominant mechanism in our system.

\begin{tabular}{|l|l|l|l|}
\hline Critical Stress $(\mathrm{GPa})$ & Fixed $(\mathrm{k}=0.5)$ & Free Rotation $(\mathrm{k}=0.7)$ & Free Translation $(\mathrm{k}=1)$ \\
\hline 0.3 & 96.0 & 68.6 & 48.0 \\
\hline 1.0 & 52.6 & 37.4 & $\mathbf{2 6 . 5}$ \\
\hline 1.3 & 46.1 & 33.0 & $\mathbf{2 3 . 0}$ \\
\hline
\end{tabular}

Table SI5: Specific aspect ratios required for critical buckling

In order for buckling to explain the range of values seen during localized failure with the free translation condition, some nanowires would need to be either $10.4 \mu \mathrm{m}$ taller than average (a 260\% increase) or $134 \mathrm{~nm}$ thinner than average (a $44 \%$ decrease). For the free rotation condition, nanowires with dimensions either $16.6 \mu \mathrm{m}$ taller than average (a $415 \%$ increase) or $183.4 \mathrm{~nm}$ thinner than average (a $61 \%$ decrease) would need to be present. All these values are far outside the observed range of $301+/-31 \mathrm{~nm}$ and $4.00+/-0.15 \mu \mathrm{m}$.

6. Calculation of tilt angle and flaw size impact on failure stress

In evaluating the impact of angle on failure stress we look at the relationship between flaw size at first failure, a, the stress at failure initiation, and the angle, $\theta$, described in equation 4 from the Results and Discussion section through the following relationship:

$$
a=\frac{K_{I C}{ }^{2} b^{2} h^{2}}{12(\sigma A \sin \theta)^{2}}-\frac{h^{2}}{3}
$$

where $\mathrm{b}$ is the nanowire thickness for $300 \mathrm{~nm}, \mathrm{~h}$ is the interfacial thickness of $5 \AA$, $K_{I C}$ is the interfacial toughness of $0.58 \mathrm{MPa} \sqrt{\mathrm{m}}$, and $\mathrm{A}$ is the area of the top surface of a $2 \mu \mathrm{m}$ diameter bundle. If we assume the same flaw distribution in order to isolate the effect of simply the angle 
on failure stress, a will take on the same value in both bundles as they will both have the same largest critical flaw size for initiation of failure. Looking at a variation in $\theta$ of $1^{\circ}$ in two bundles we show the maximum impact of the variation using a flaw size of $300 \mathrm{~nm}$ and tilt angles of $2^{\circ}$ and $3^{\circ}$, Solving for stress we see that the stress of failure initiation is $1.3 \mathrm{GPa}$ for the bundle with $2^{\circ}$ tilt and $0.87 \mathrm{GPa}$ for the bundle with $3^{\circ}$ tilt. This represents an upper bound of a $0.43 \mathrm{GPa}$ difference in initial failure stress.

For comparing the impact of flaw size on failure stress observed, we use the equation above but with a fixed failure stress and identify the crack length associated with a particular tilt angle. We determine that both a $217 \mathrm{~nm}$ defect with a tilt of $3^{\circ}$ and a $163 \mathrm{~nm}$ with a tilt of $4^{\circ}$ will initiate failure at $0.7 \mathrm{GPa}$.

7. Derivation of probability function for failure

Probability that an element of the bundle can survive is $\check{f}$. The initiation rate of a particular failure can be describes as the fractional reduction rate of intact elements within the next time interval.

$$
\dot{n}_{i}=-\frac{1}{\check{f}_{i}} \frac{d \check{f}_{i}}{d t}
$$

looking along a common strain path,

$$
\ln \left(\check{f}_{i}\right)=-\int \frac{\dot{n}_{i}}{\dot{\varepsilon}} d L
$$

which is equivalent to

$$
\ln (\check{f})=-\int \frac{\sum \dot{n}}{\dot{\varepsilon}} d \varepsilon
$$


The initiation rate can also be defined by the probability of a particular element reaching the failure strain of the material or failure mechanism

$$
\dot{n}_{i}=v d N_{i} \exp \left[-\left(\frac{\varepsilon_{c}}{\varepsilon}\right)^{m}\right]
$$

with $\varepsilon_{c}$ being equal to the critical failure strain as a function of $\varepsilon$ being the current strain. $m$ is the Weibull exponent and relates to the failure prediction of the material or mechanism. $v$ is the attempt frequency. Combining this definition with the above equation for $\check{f}$ gives

$$
\ln (\check{f})=-v \iint \frac{1}{\dot{\varepsilon}} \exp \left[-\left(\frac{\varepsilon_{c}}{\varepsilon}\right)^{m}\right] d N d \varepsilon
$$

The probability of failure can be defined as $f=1-\check{f}$ and thus

$$
f=1-\exp \left[-v \iint \frac{1}{\dot{\varepsilon}} \exp \left[-\left(\frac{\varepsilon_{c}}{\varepsilon}\right)^{m}\right] d N d \varepsilon\right]
$$

Further manipulation of this equation assuming linear elasticity gives rise to a solution assuming uniform strain in compression of the structure and assuming a constant strain rate.

$$
f(\sigma)=1-\exp \left[\frac{v N}{\dot{\sigma}} \int_{0}^{\sigma} \exp \left[-\left(\frac{\sigma_{c}}{\sigma^{\prime}}\right)^{m}\right] d \sigma^{\prime}\right]
$$

with $\dot{\sigma}$ as the stress loading rate, $v$ as the attempt frequency for failure, $\mathrm{N}$ as the total number of nanowires, $\sigma c$ as the critical failure stress, and $\mathrm{m}$ as the Weibull exponent. The $v / \dot{\sigma}$ ratio ultimately determines the "stress step size" over which failure attempts occur. 